\title{
Cyclotriphosphazenes as scaffolds for the synthesis of metallomesogens
}

Josefina Jiménez, *[a] José Antonio Sanz, ${ }^{[a]}$ José Luis Serrano, ${ }^{[b, c]}$ Joaquín Barberá, ${ }^{[c]}$ Luis Oriol [c]

[a] Dr. J. Jiménez, J.A. Sanz

Departamento de Química Inorgánica, Facultad de Ciencias - Instituto de Síntesis Química y Catálisis Homogénea (ISQCH). Universidad de Zaragoza-CSIC. Zaragoza 50009. Spain. Email: jiimvil@unizar.es

[b] Prof. J.L. Serrano.

Departamento de Química Orgánica, Facultad de Ciencias - Instituto Universitario de Nanociencia de Aragón (INA). Universidad de Zaragoza. Zaragoza 50018. Spain.

[c] Prof. J. Barberá, Prof. L. Oriol

Departamento de Química Orgánica, Facultad de Ciencias - Instituto de Ciencia de Materiales de Aragón (ICMA). Universidad de ZaragozaCSIC. Zaragoza 50009. Spain.

\section{SUPPORTING INFORMATION}

Table of Contents:

1. Synthesis and characterization details of isocyanide ligand, $L$.

2. ${ }^{31} \mathrm{P}\left\{{ }^{1} \mathrm{H}\right\}$ NMR spectrum of compound phos-2.1 in $\mathrm{CD}_{2} \mathrm{Cl}_{2}$ at $-80^{\circ} \mathrm{C}$ (Figure S1).

3. DSC scans for all compounds phos-1.n ( $n=1,2$, or 3$)$ and phos-2.n $(n=1$, or 2) (Figures S2-S6).

4. X-ray diffractograms of the precursor $[\mathrm{Ag}(\mathrm{OTf}) \mathrm{L}]$ and derived mesomorphic compounds phos-1.3 and phos-2.3 (Figures S7, S8, and S9, respectively).

1. Synthesis and characterization details of isocyanide ligand, L.

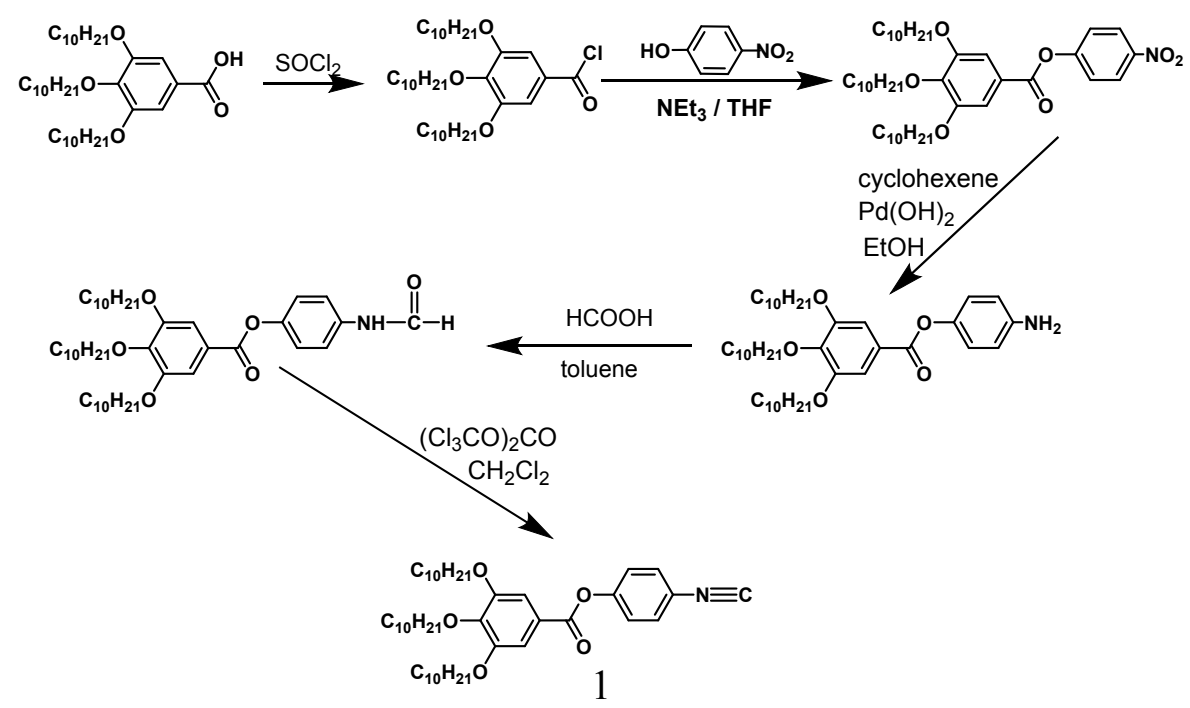


Synthesis of $\left(\mathrm{NO}_{2}\right) \mathrm{C}_{6} \mathrm{H}_{4}\left\{\mathrm{OC}(\mathrm{O}) \mathrm{C}_{6} \mathrm{H}_{2}\left(3,4,5-\left(\mathrm{OC}_{10} \mathrm{H}_{21}\right)_{3}\right)\right\}-4$. To a solution of $\mathrm{CIC}(\mathrm{O}) \mathrm{C}_{6} \mathrm{H}_{2}\left(3,4,5-\left(\mathrm{OC}_{10} \mathrm{H}_{21}\right)_{3}(6.094 \mathrm{~g}, 10 \mathrm{mmol})\right.$ in dry tetrahydrofuran $(75 \mathrm{~mL})$ under an argon atmosphere, 4-nitrophenol $(1.391 \mathrm{~g}, 10 \mathrm{mmol})$ and excess of $\mathrm{NEt}_{3}(8.5 \mathrm{~mL}$, $60 \mathrm{mmol}$ ) were added. The mixture was stirred for approximately 3 days at RT and filtered off. Evaporation of the solvent gave the product a white solid, which was purify washing with ethanol $(3 \times 5 \mathrm{~mL})$ and dried in vacuo for $24 \mathrm{~h}$. Yield: $6.41 \mathrm{~g}, 90 \%$

${ }^{1} \mathrm{H}$ NMR $\left(\left(\mathrm{CD}_{3}\right)_{2} \mathrm{CO}\right): \delta=8.38$ ("d", $\left.{ }^{3} \mathrm{~J}(\mathrm{H}, \mathrm{H})=8.8 \mathrm{~Hz}, 2 \mathrm{H} ;\left(\mathrm{NO}_{2}\right) \mathrm{C}_{6} \mathrm{H}_{4} \mathrm{O}\right), 7.60$ ("d", $\left.{ }^{3} \mathrm{~J}(\mathrm{H}, \mathrm{H})=8.8 \mathrm{~Hz}, 2 \mathrm{H} ;\left(\mathrm{NO}_{2}\right) \mathrm{C}_{6} \mathrm{H}_{4} \mathrm{O}\right), 7.47\left(\mathrm{~s}, 2 \mathrm{H} ; \mathrm{C}_{6} \mathrm{H}_{2}\left(\mathrm{OC}_{10} \mathrm{H}_{21}-p\right)_{3}\right), 4.11$ ("t", ${ }^{3} J(\mathrm{H}, \mathrm{H})=$ $\left.6.3 \mathrm{~Hz}, 4 \mathrm{H} ; \mathrm{OCH}_{2}\right), 4.09$ ("t", $\left.3 \mathrm{~J}(\mathrm{H}, \mathrm{H})=6.5 \mathrm{~Hz}, 2 \mathrm{H} ; \mathrm{OCH}_{2}\right), 1.84\left(\mathrm{~m}, 4 \mathrm{H} ; \mathrm{CH}_{2}\right), 1.76(\mathrm{~m}$, $\left.2 \mathrm{H} ; \mathrm{CH}_{2}\right), 1,55\left(\mathrm{~m}, 6 \mathrm{H} ; \mathrm{CH}_{2}\right), 1.42-1.29\left(\mathrm{~m}, 36 \mathrm{H} ; \mathrm{CH}_{2}\right), 0.88\left(\mathrm{~m}, 9 \mathrm{H} ; \mathrm{CH}_{3}\right)$.

Synthesis of $\left(\mathrm{NH}_{2}\right) \mathrm{C}_{6} \mathrm{H}_{4}\left\{\mathrm{OC}(\mathrm{O}) \mathrm{C}_{6} \mathrm{H}_{2}\left(3,4,5-\left(\mathrm{OC}_{10} \mathrm{H}_{21}\right)_{3}\right)\right\}-4$. To a solution of $\left(\mathrm{NO}_{2}\right) \mathrm{C}_{6} \mathrm{H}_{4}\left\{\mathrm{OC}(\mathrm{O}) \mathrm{C}_{6} \mathrm{H}_{2}\left(3,4,5-\left(\mathrm{OC}_{10} \mathrm{H}_{21}\right)_{3}\right)\right\}-4(2.136 \mathrm{~g}, 3 \mathrm{mmol})$ in ethanol $(30 \mathrm{~mL})$ under an argon atmosphere, cyclohexene $(32 \mathrm{~mL})$ and palladium hydroxide $(20 \mathrm{wt} \%$ on carbon, $0.2 \mathrm{~g}$ ) were added. The mixture was refluxed for approximately $8 \mathrm{~h}$ and filtered. The solvent was evaporated giving a pale-yellow oil, which was recrystallized in acetone and dried in vacuo for $24 \mathrm{~h}$. Yield: $1.290 \mathrm{~g}, 63 \%$.

${ }^{1} \mathrm{H}$ NMR ((CD $\left.)_{2} \mathrm{CO}\right): \delta=7.40$ (s, $\left.2 \mathrm{H} ; \mathrm{C}_{6} \mathrm{H}_{2}\left(\mathrm{OC}_{10} \mathrm{H}_{21}-p\right)_{3}\right), 6.92$ ("d", ${ }^{3} J(\mathrm{H}, \mathrm{H})=8.8 \mathrm{~Hz}$, $2 \mathrm{H}$; $\left(\mathrm{NH}_{2}\right) \mathrm{C}_{6} \mathrm{H}_{4} \mathrm{O}$ ), 6.71 ("d", ${ }^{3} \mathrm{~J}(\mathrm{H}, \mathrm{H})=8.8 \mathrm{~Hz}, 2 \mathrm{H}$; $\left.\left(\mathrm{NH}_{2}\right) \mathrm{C}_{6} \mathrm{H}_{4} \mathrm{O}\right), 4.64\left(\mathrm{br}, 2 \mathrm{H} ; \mathrm{NH}_{2}\right)$, 4.09 ("t", $\left.{ }^{3} J(\mathrm{H}, \mathrm{H})=6.3 \mathrm{~Hz}, 4 \mathrm{H} ; \mathrm{OCH}_{2}\right), 4.06$ ("t", $\left.{ }^{3} \mathrm{~J}(\mathrm{H}, \mathrm{H})=6.3 \mathrm{~Hz}, 2 \mathrm{H} ; \mathrm{OCH}_{2}\right), 1.84(\mathrm{~m}$, $\left.4 \mathrm{H} ; \mathrm{CH}_{2}\right), 1.76\left(\mathrm{~m}, 2 \mathrm{H} ; \mathrm{CH}_{2}\right), 1,55\left(\mathrm{~m}, 6 \mathrm{H} ; \mathrm{CH}_{2}\right), 1.41-1.25\left(\mathrm{~m}, 36 \mathrm{H} ; \mathrm{CH}_{2}\right), 0.89(\mathrm{~m}, 9 \mathrm{H}$; $\mathrm{CH}_{3}$ ).

Synthesis of $\{\mathrm{HC}(\mathrm{O}) \mathrm{NH}\} \mathrm{C}_{6} \mathrm{H}_{4}\left\{\mathrm{OC}(\mathrm{O}) \mathrm{C}_{6} \mathrm{H}_{2}\left(3,4,5-\left(\mathrm{OC}_{10} \mathrm{H}_{21}\right)_{3}\right)\right\}-4$. A flask fitted with a Dean-Stark apparatus was charged with a solution of $\left(\mathrm{NH}_{2}\right) \mathrm{C}_{6} \mathrm{H}_{4}\left\{\mathrm{OC}(\mathrm{O}) \mathrm{C}_{6} \mathrm{H}_{2}(3,4,5-\right.$ $\left.\left.\left(\mathrm{OC}_{10} \mathrm{H}_{21}\right)_{3}\right)\right\}-4(2.046 \mathrm{~g}, 3 \mathrm{mmol})$ in $60 \mathrm{~mL}$ of toluene. Formic acid (12 mL, 98\%) was added and the resulting solution was refluxed for $2 \mathrm{~h}$ and then cooled to room temperature. The solvent was removed to give the product as a white solid, which was dried in vacuo for $24 \mathrm{~h}$. Yield: $1.205 \mathrm{~g}, 56.6 \%$.

${ }^{1} \mathrm{H}$ NMR $\left(\left(\mathrm{CD}_{3}\right)_{2} \mathrm{CO}\right): \delta=8.22(\mathrm{~s}, 1 \mathrm{H} ; \mathrm{C}(\mathrm{O}) \mathrm{H}), 7.76$ ("d", $\left.{ }^{3} \mathrm{~J}(\mathrm{H}, \mathrm{H})=8.9 \mathrm{~Hz}, 2 \mathrm{H} ; \mathrm{C}_{6} \mathrm{H}_{4} \mathrm{O}\right)$, 7.42 (s, 2H; $\left.\mathrm{C}_{6} \mathrm{H}_{2}\left(\mathrm{OC}_{10} \mathrm{H}_{21}-p\right)_{3}\right), 7.21$ ("d", $\left.{ }^{3} \mathrm{~J}(\mathrm{H}, \mathrm{H})=8.9 \mathrm{~Hz}, 2 \mathrm{H} ; \mathrm{C}_{6} \mathrm{H}_{4} \mathrm{O}\right), 4.09$ ("t", ${ }^{3} \mathrm{~J}(\mathrm{H}, \mathrm{H})=6.3 \mathrm{~Hz}, 4 \mathrm{H} ; \mathrm{OCH}_{2}$ ), 4.06 ("t", $\left.{ }^{3} \mathrm{~J}(\mathrm{H}, \mathrm{H})=6.4 \mathrm{~Hz}, 2 \mathrm{H} ; \mathrm{OCH}_{2}\right), 2.85(\mathrm{br}, 1 \mathrm{H} ; \mathrm{NH})$, $1.84\left(\mathrm{~m}, 4 \mathrm{H} ; \mathrm{CH}_{2}\right), 1.76\left(\mathrm{~m}, 2 \mathrm{H} ; \mathrm{CH}_{2}\right), 1,54\left(\mathrm{~m}, 6 \mathrm{H} ; \mathrm{CH}_{2}\right), 1.42-1.29\left(\mathrm{~m}, 36 \mathrm{H} ; \mathrm{CH}_{2}\right)$, $0.88\left(\mathrm{~m}, 9 \mathrm{H} ; \mathrm{CH}_{3}\right)$. 
Synthesis of $\left\{\mathrm{CN} \mathrm{C}_{6} \mathrm{H}_{4}\left\{\mathrm{OC}(\mathrm{O}) \mathrm{C}_{6} \mathrm{H}_{2}\left(3,4,5-\left(\mathrm{OC}_{10} \mathrm{H}_{21}\right)_{3}\right)\right\}-4\right.$. To a solution of $\{\mathrm{HC}(\mathrm{O}) \mathrm{NH}\} \mathrm{C}_{6} \mathrm{H}_{4}\left\{\mathrm{OC}(\mathrm{O}) \mathrm{C}_{6} \mathrm{H}_{2}\left(3,4,5-\left(\mathrm{OC}_{10} \mathrm{H}_{21}\right)_{3}\right)\right\}-4 \quad(1.00 \mathrm{~g}, 1.41 \mathrm{mmol})$ and triethylamine $(0.45 \mathrm{~mL}, 3.1 \mathrm{mmol}, 10 \%$ excess $)$ in $40 \mathrm{~mL}$ of $\mathrm{CH}_{2} \mathrm{Cl}_{2}$ was added dropwise a solution of triphosgene $(0.154 \mathrm{~g}, 0.52 \mathrm{mmol}, 10 \%$ excess) in $40 \mathrm{~mL}$ of $\mathrm{CH}_{2} \mathrm{Cl}_{2}$. The mixture was stirred for $1 \mathrm{~h}$ and then the solvent was removed on a rotary evaporator. The resulting residue was chromatographed (silica gel, $\mathrm{CH}_{2} \mathrm{Cl}_{2}$-hexane, 3:1 as eluent) and the solvent was evaporated to obtain the product as a white solid, and dried in vacuo for $24 \mathrm{~h}$. $(0.578 \mathrm{~g}, 59.2 \%)$.

2. ${ }^{31} \mathrm{P}\left\{{ }^{1} \mathrm{H}\right\}$ NMR spectrum of compound phos- -1.1 in $\mathrm{CD}_{2} \mathrm{Cl}_{2}$ at $-80^{\circ} \mathrm{C}$ (Figure $\mathrm{S} 1$ ).

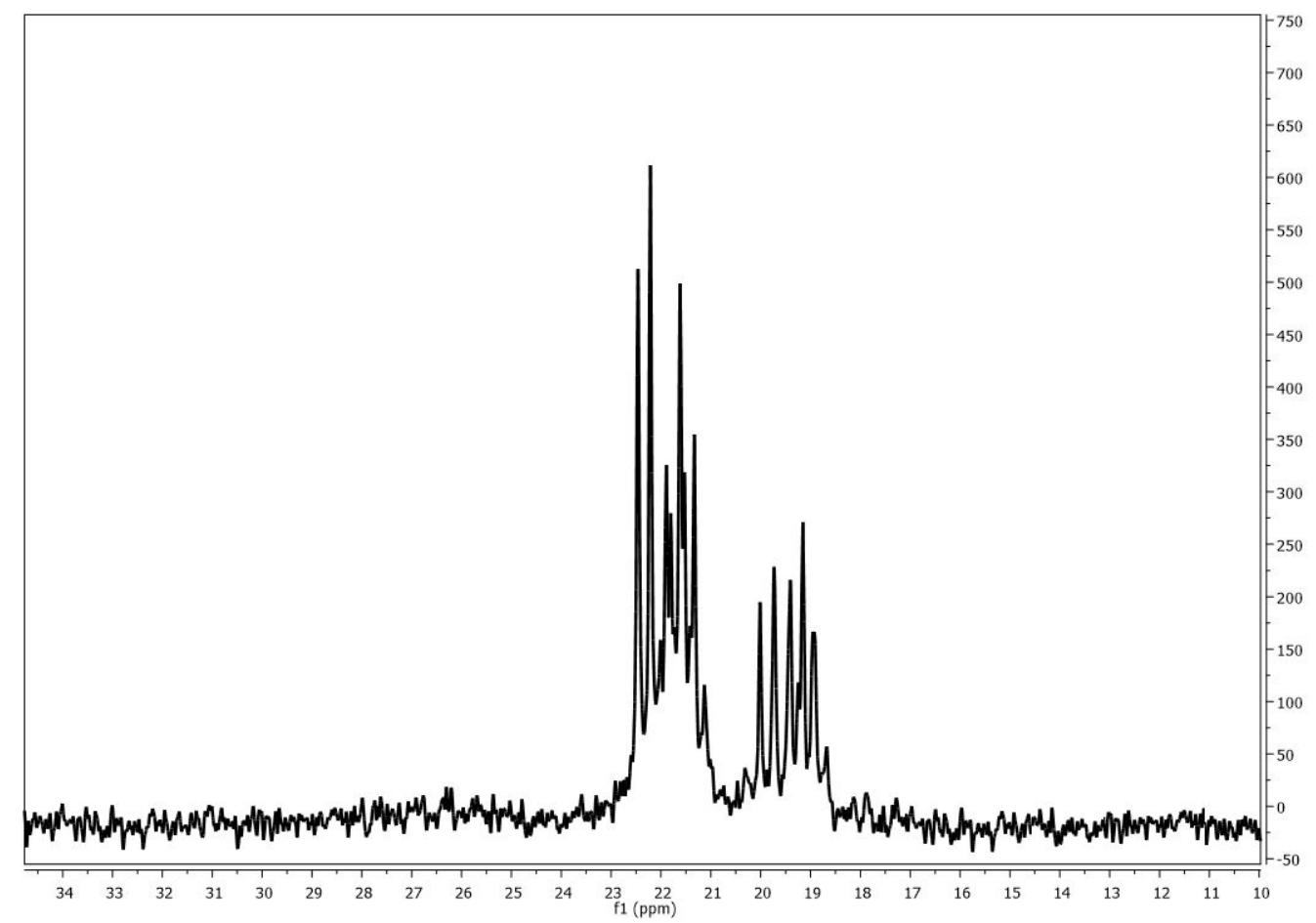

Figure S1. ${ }^{31} \mathrm{P}\left\{{ }^{1} \mathrm{H}\right\}$ NMR spectrum of compound phos-2.1 in $\mathrm{CD}_{2} \mathrm{Cl}_{2}$ at $-80^{\circ} \mathrm{C}$ 
3. DSC scans for all compounds phos-1. $n$ and phos-2. $(n=1,2$, or 3$)$ (Figures S2-S6).

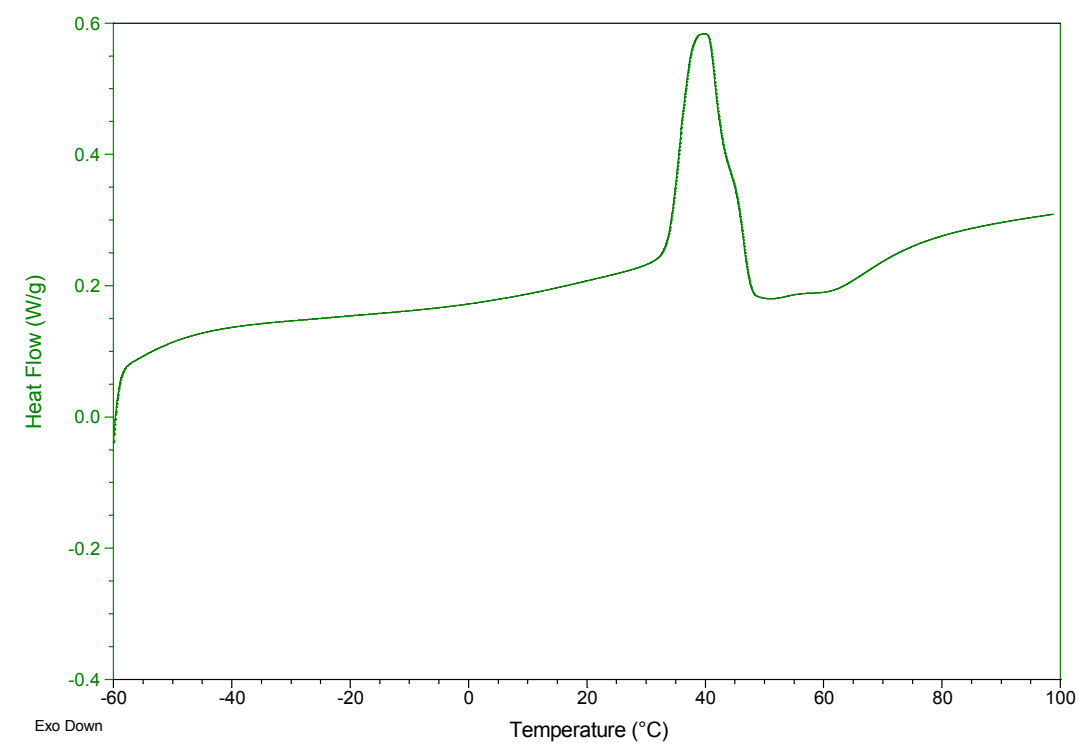

Figure S2. DSC first heating at $10^{\circ} \mathrm{C} / \mathrm{min}$ of phos-1.1. Decomposition is observed upon melting as an exothermic peak. Subsequent scans are not reproducible.

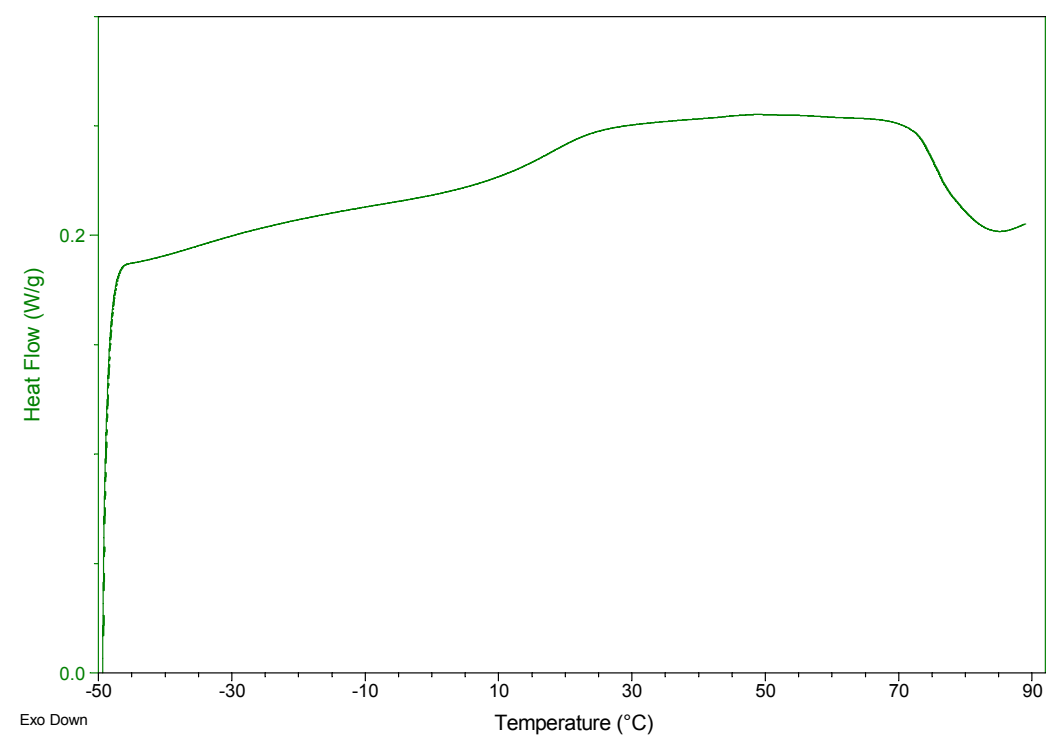

Figure S3. DSC first heating at $10^{\circ} \mathrm{C} / \mathrm{min}$ of phos-1.2. Decomposition is observed above $\mathrm{Tg}$ as an exothermic peak at around $70^{\circ} \mathrm{C}$. Subsequent scans are not reproducible. 


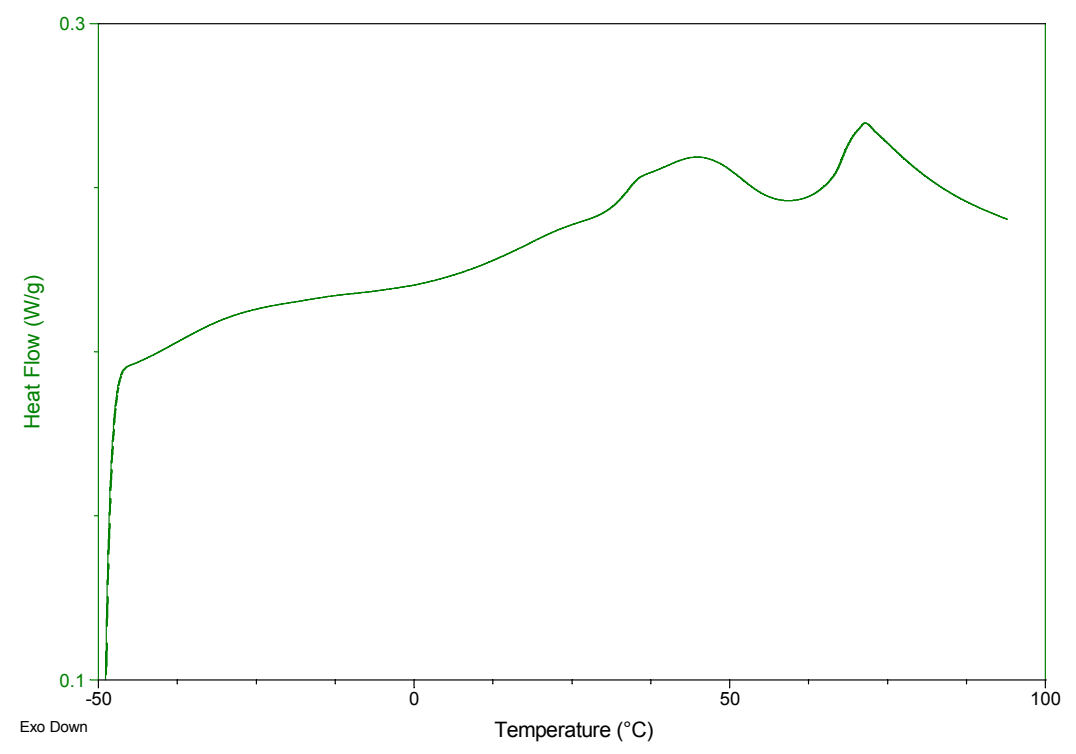

Figure S4. DSC first heating at $10^{\circ} \mathrm{C} / \mathrm{min}$ of phos-1.3. Decomposition is observed above aprox. $70^{\circ} \mathrm{C}$. Subsequent scans are not reproducible.

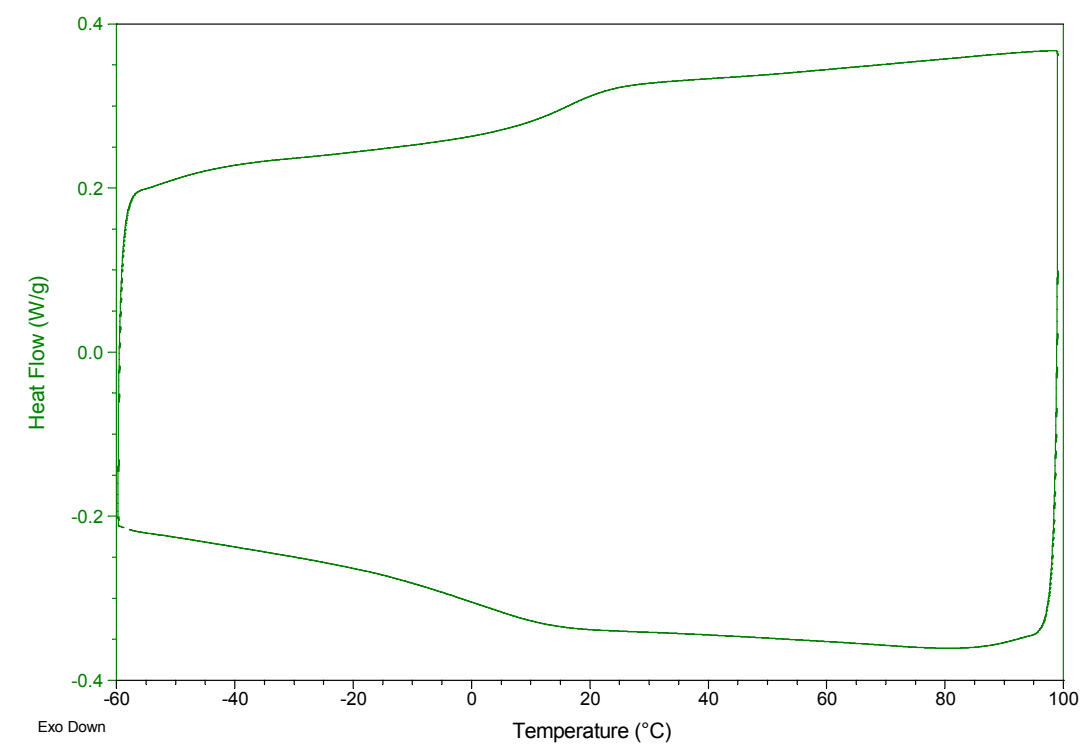

Figure S5. DSC second heating (top) and cooling (down) scans at $10^{\circ} \mathrm{C} / \mathrm{min}$ of phos-2.1. 


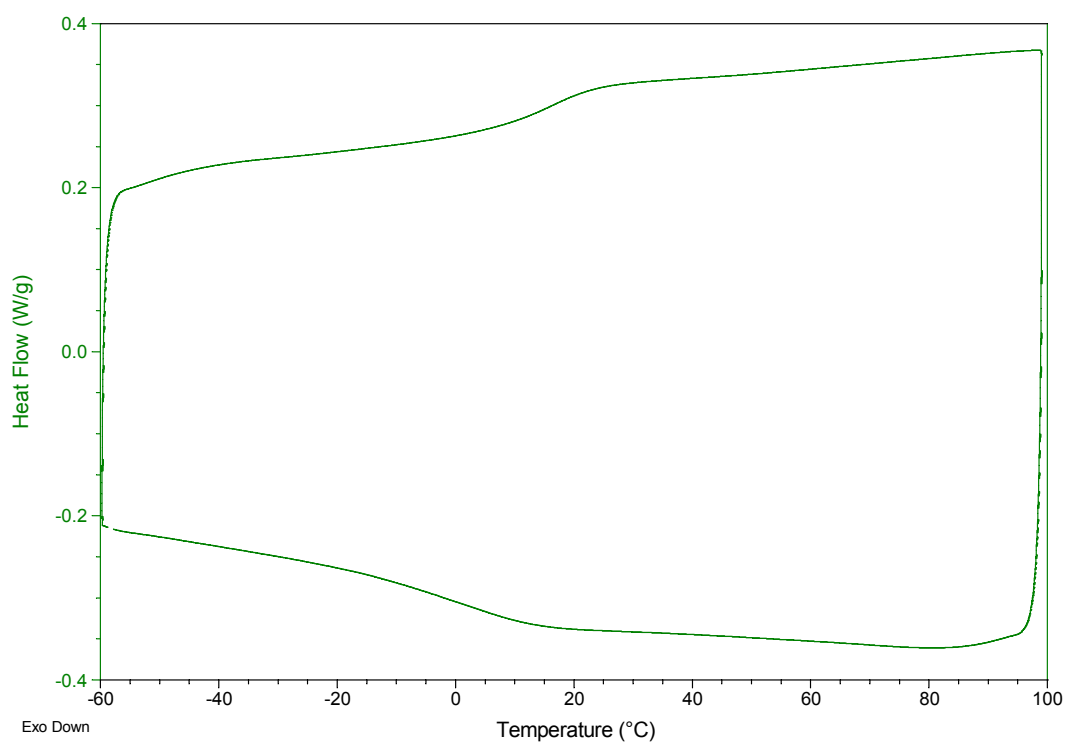

Figure S6. DSC second heating (top) and cooling (down) scans at $10^{\circ} \mathrm{C} / \mathrm{min}$ of phos-2.2.

4. X-ray diffractograms of the precursor $[\mathrm{Ag}(\mathrm{OTf}) \mathrm{L}]$ and derived mesomorphic compounds phos-1.3 and phos-2.3 (Figures S7, S8, and S9, respectively).

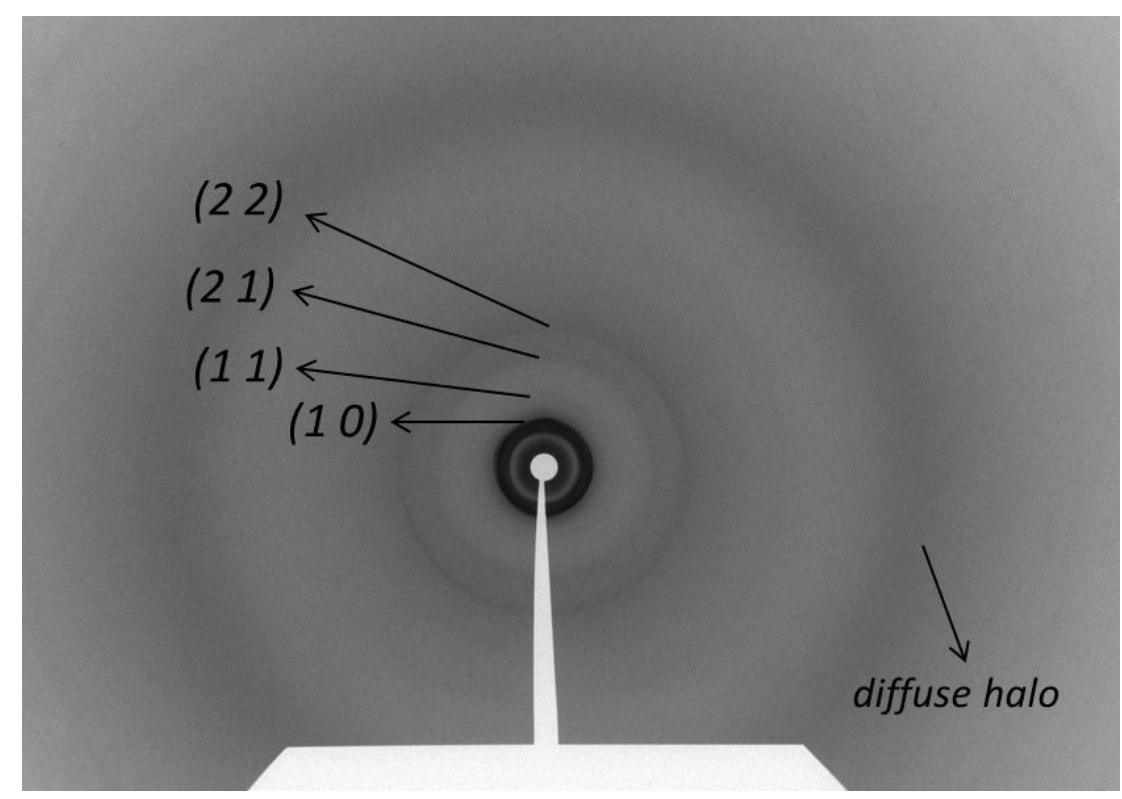

Figure S7. X-ray diffractogram of [Ag(OTf)CNR] taken at room temperature and proposed indexation. The (2 2 ) reflection is very weak, but visible in the original pattern. 


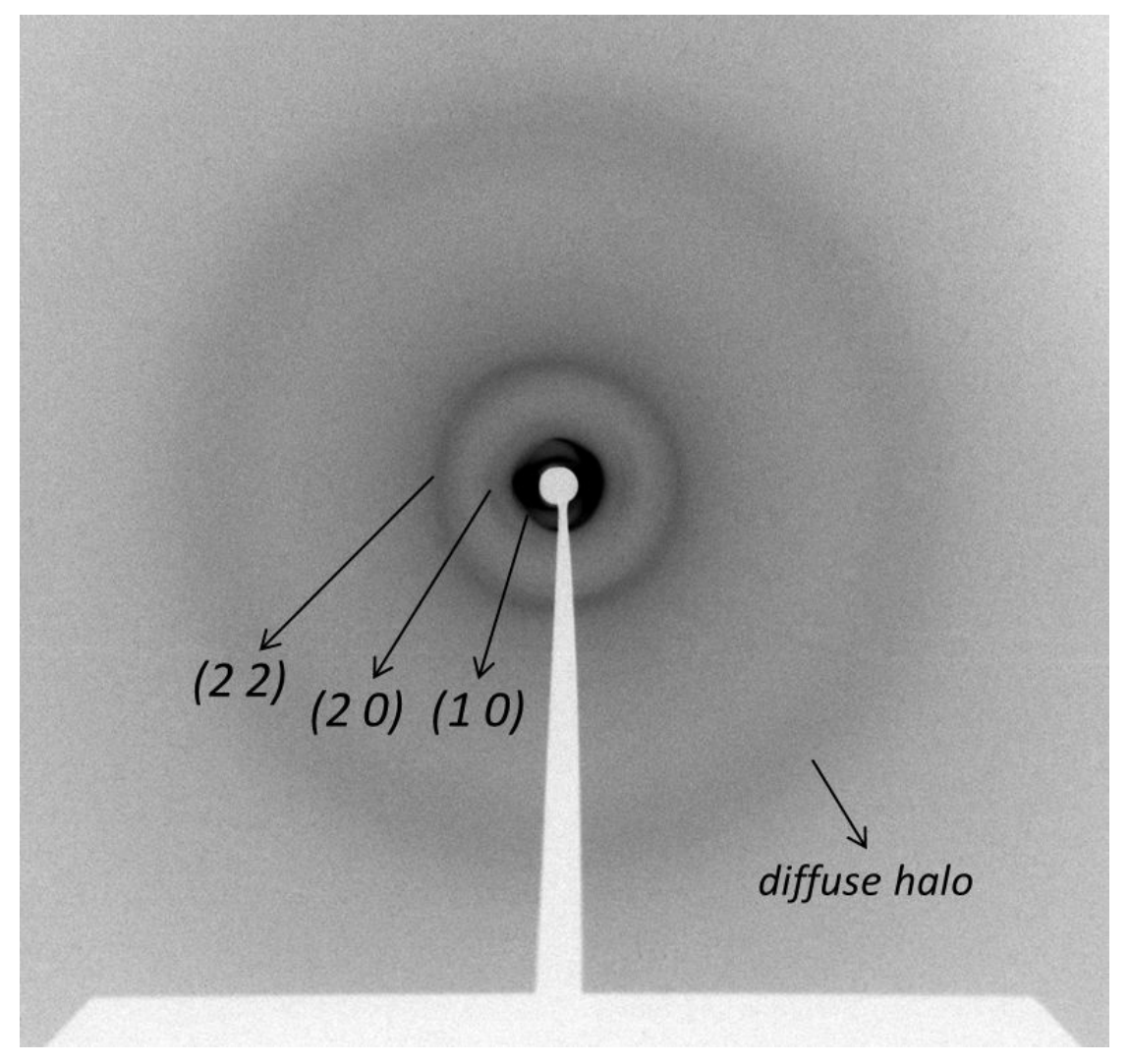

Figure S8. X-ray diffractogram of phos-1.3 taken at room temperature and proposed indexation.

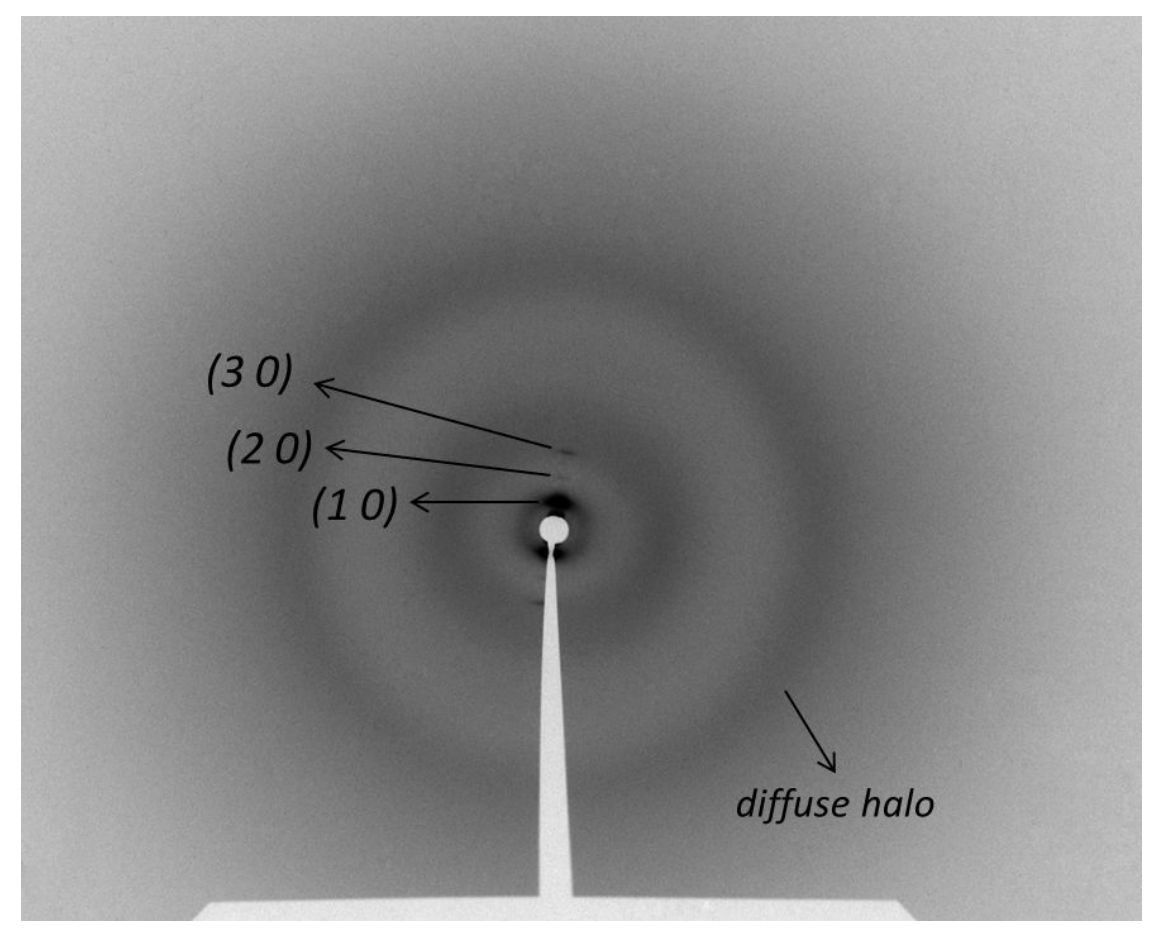

Figure S9. X-ray diffractogram of an aligned sample of phos-2.3 taken at room temperature and proposed indexation. 